\title{
Síndrome de Fisher-Evans
}

asociado a pancreatitis aguda

Fisher-Evans syndrome associated to acute pancreatitis

Síndrome de Fisher-Evans associada a pancreatite aguda

Rafael Domínguez-Pérez, * Fernando Valentín Arrazola-García*

\section{RESUMEN}

El síndrome de Fisher-Evans o de Evans es la manifestación de anemia hemolítica inmunitaria que puede ser simultánea o subsecuente a trombocitopenia inmunitaria. Debido a que su diagnóstico no es común, en la actualidad, no hay cifras exactas acerca de su prevalencia. De acuerdo con su origen, puede ser un evento idiopático o secundario.

Presentamos el caso de un hombre de 58 años que desarrolló síndrome de Fisher-Evans asociado a pancreatitis aguda, en la Unidad de Terapia Intensiva (UTI). Caracterizado por anemia hemolítica inmunitaria (Coombs positivo) y trombocitopenia; además presentó disfunción renal.

Durante su estancia hospitalaria, recibió tratamiento con esteroide; metilprednisolona intravenosa por tres días, prednisona vía oral (1 mg/kg/día) e inmunoglobulina humana $(0.4 \mathrm{~g} / \mathrm{kg} / \mathrm{día})$ por cinco días.

Resultados: Presentó mejoría del cuadro clínico, con resolución de la anemia y trombocitopenia. Fue valorado por el Servicio de Nefrología; que instauró manejo con hemodiálisis y realizó biopsia renal con reporte de necrosis tubular intersticial. Conclusión: La detección temprana de dicho síndrome y el manejo oportuno tuvo gran impacto en la evolución clínica del paciente. Fue egresado a domicilio y recibió seguimiento por parte de los servicios de nefrología y hematología por consulta externa.

Palabras clave: Síndrome de Fisher-Evans, pancreatitis, unidad de terapia intensiva.

\section{ABSTRACT}

Fisher-Evans syndrome or Evans syndrome is the manifestation of immune hemolytic anemia that may be simultaneous or subsequent to immune thrombocytopenia. Because its diagnosis is not common, there are currently no exact information about its prevalence. According to its origin, it can be an idiopathic or secondary event.

We present the case of a 58-year-old male, who developed Fisher-Evans syndrome associated to acute pancreatitis, in the intensive care unit (ICU); characterized by immune hemolytic anemia (Coombs positive) and thrombocytopenia; also presented renal dysfunction.

During his hospital stay, he received steroid treatment; intravenous methylprednisolone for three days, oral prednisone $(1 \mathrm{mg} / \mathrm{kg} /$ day $)$ and human immunoglobulin $(0.4 \mathrm{~g} / \mathrm{kg} /$ day) for five days.

Results: He presented clinical improvement, with resolution of anemia and thrombocytopenia. It was evaluated by the nephrology service; who established management with hemodialysis and performed renal biopsy with a report of interstitial tubular necrosis.

Conclusion: Early detection of this syndrome and timely management had a great impact on the clinical evolution of the patient. He was graduated from home and received follow-up by the nephrology and hematology services by external. Keywords: Fisher-Evans syndrome, pancreatitis, intensive care unit.

\section{RESUMO}

A síndrome de Fisher-Evans ou Evans é a manifestação de anemia hemolítica imune que pode ser simultânea ou subsequente à trombocitopenia imune. Como seu diagnóstico não é comum, atualmente não há números exatos sobre sua prevalência. De acordo com sua origem, pode ser um evento idiopático ou secundário.

Apresentamos o caso de um homem de 58 anos que desenvolveu a síndrome de Fisher-Evans associada a pancreatite aguda, internado em Unidade de

\footnotetext{
* Unidad de Cuidados Intensivos Adultos. Unidad de Terapia Intensiva. Hospital General Regional No. 1 «Dr. Carlos Mac Gregor Sánchez Navarro». IMSS.

Recibido: 10/10/2019. Aceptado: 23/12/2020

Citar como: Domínguez-Pérez R, Arrazola-García FV. Síndrome de FisherEvans asociado a pancreatitis aguda. Med Crit. 2021;35(3):154-156. https://dx.doi. org/10.35366/100005

www.medigraphic.com/medicinacritica
}

Terapia Intensiva (UTI). Caracterizada por anemia hemolítica imunológica (Coombs positivo) e trombocitopenia; además o paciente apresentava disfunção renal.

Durante sua internação, ele recebeu tratamento com esteróides; metilprednisolona intravenosa por 3 dias, prednisona oral (1 $\mathrm{mg} / \mathrm{kg} / \mathrm{dia})$ e imunoglobulina humana $(0.4 \mathrm{~g} / \mathrm{kg} / \mathrm{dia})$ por 5 dias.

Resultados: Apresentou melhora do quadro clínico, com resolução da anemia e trombocitopenia. Foi avaliado pelo serviço de nefrologia; que instituiu tratamento com hemodiálise e realizou biópsia renal com relato de necrose tubular intersticial.

Conclusão: A detecção precoce desta síndrome e o manejo oportuno tiveram grande impacto na evolução clínica do paciente. Recebeu alta hospitalar e acompanhamento nos serviços de nefrologia e hematologia em consulta ambulatorial.

Palavras-chave: Síndrome de Fisher-Evans, pancreatite, Unidade de Terapia Intensiva.

\section{INTRODUCCIÓN}

El síndrome de Fisher-Evans o de Evans es la manifestación de anemia hemolítica inmunitaria que puede ser simultánea o subsecuente a trombocitopenia inmunitaria. Debido a que su diagnóstico no es común, en la actualidad no hay cifras exactas acerca de su prevalencia en todo el mundo; se estima que de 0.8 a $4 \%$ de los pacientes con trombocitopenia inmunitaria o anemia hemolítica inmunitaria padecen este síndrome. ${ }^{1}$ De acuerdo con su origen, puede ser un evento idiopático o secundario; aproximadamente $50 \%$ es del primer tipo y del grupo secundario, $41 \%$ se asocia con enfermedades autoinmunitarias (de manera más frecuente con lupus eritematoso sistémico), $17 \%$ se asocia con inmunodeficiencias y con linfomas. ${ }^{2}$ Se han reportado casos en la literatura de este síndrome asociados a esclerodermia, síndrome de anticuerpos antifosfolípidos y al embarazo, entre otras causas. ${ }^{3,4}$

Aunque en $2 \%$ de los pacientes con púrpura trombocitopénica trombótica (PTT) se desarrolla inflamación pancreática, la PTT secundaria a pancreatitis aguda es un hecho muy infrecuente; en el curso de una pancreatitis se desarrolla principalmente entre las primeras 48 y 72 horas tras el inicio de los síntomas. ${ }^{5}$

El tratamiento de primera línea para el síndrome de Fisher-Evans es, por lo general, con corticosteroides y/o inmunoglobulina intravenosa, a lo que la mayoría de los pacientes responden; sin embargo, el fracaso al tratamiento es frecuente. Las opciones para el tratamiento de segunda línea incluyen medicamentos inmunosupresores; ciclosporina, micofenolato de mo- 
fetilo, vincristina, danazol o una combinación de estos fármacos. ${ }^{6}$

\section{PRESENTACIÓN DEL CASO}

Hombre de 58 años, con consumo crónico de alcohol 20 gramos al día desde los 20 años. Consumo de nux vomica (nuez vómica) y sulfur (azufre). Inició padecimiento el 15 de octubre de 2019 con dolor torácico opresivo, dolor abdominal en epigastrio y diaforesis, se trasladó a urgencias, donde presentó lesión renal aguda, elevación de bilirrubinas totales y se sospechó falla hepática aguda, motivo por el que ingresó a la Unidad de Terapia Intensiva (UTI).

En la UTI se diagnosticó pancreatitis aguda, con base en criterios de Atlanta; elevación de enzimas pancreáticas, lipasa $>1,400 \mathrm{UI} / \mathrm{L}$ y estudio tomográfico de abdomen, donde se estadificó como pancreatitis aguda Balthazar C (Figura 1), con datos de severidad por presencia de datos de disfunción orgánica, disfunción renal y hepática.

El segundo día en la Unidad de Terapia Intensiva se mantuvo hemodinámicamente estable, presentó petequias en extremidades superiores e inferiores, elevación de bilirrubinas totales, descenso de hemoglobina y trombocitopenia. Se realizó prueba de
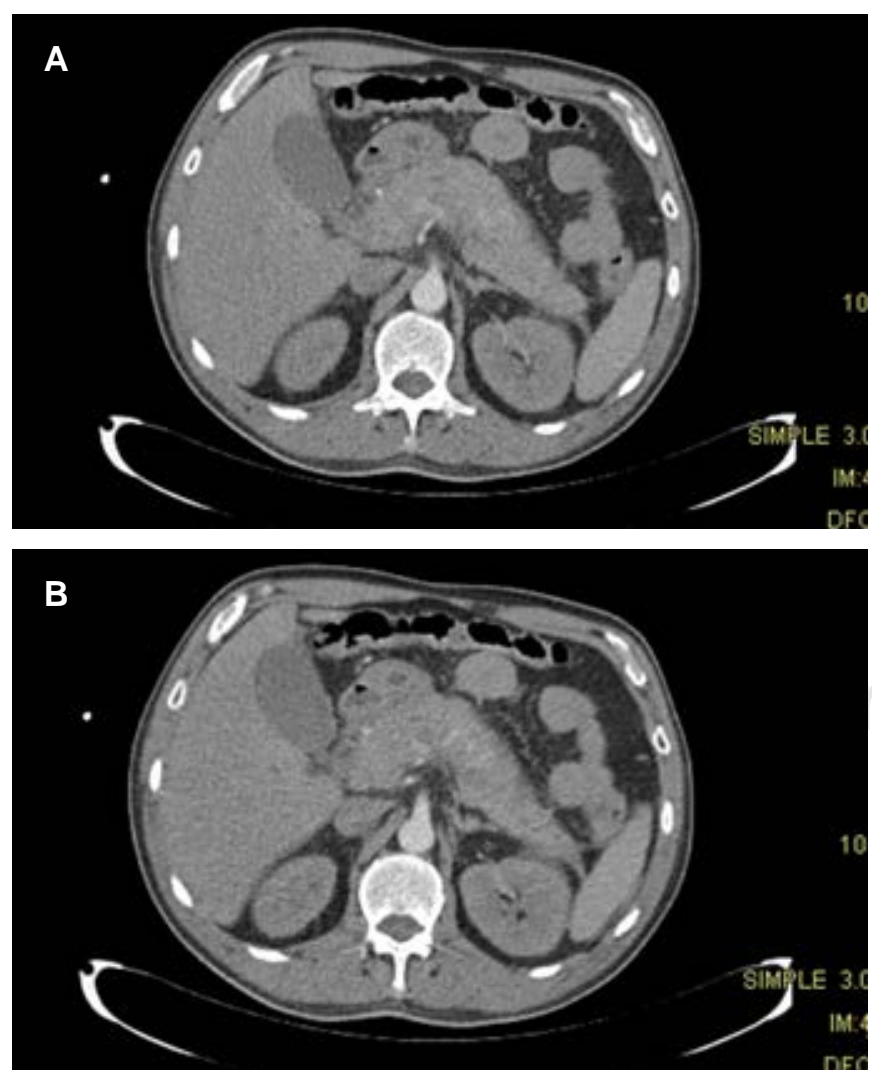

Figura 1: Imagen de tomografía axial computada que demuestra pancreatitis.
Coombs directo con resultado positivo $3+$. Por presencia de anemia hemolítica autoinmune y púrpura trombocitopénica, se diagnosticó síndrome de FisherEvans. Se inició manejo con metilprednisolona 500 mg IV cada 24 horas e inmunoglobulina humana a dosis de $0.4 \mathrm{~g} / \mathrm{kg} / \mathrm{dí}$.

Se reportó biometría hemática con leucocitos de 4,000 células $/ \mathrm{mm}^{3}$, neutrófilos 3,500 células $/ \mathrm{mm}^{3}$, linfocitos 500 células $/ \mathrm{mm}^{3}$, hemoglobina $7.2 \mathrm{~g} / \mathrm{dL}$, hematocrito $22.1 \%$, plaquetas 29,000 células $/ \mathrm{mm}^{3}$, tiempos de coagulación, TP 16 segundos, INR 1.29, TTPa 33.3 segundos. Transaminasas: AST $765 \mathrm{UI} / \mathrm{L}$, ALT $425 \mathrm{UI} / \mathrm{L}$, deshidrogenasa láctica 3,555 UI/L, proteínas totales de $4.7 \mathrm{~g} / \mathrm{dL}$. Bilirrubina total $8.29 \mathrm{mg} / \mathrm{dL}$, bilirrubina directa $4.61 \mathrm{mg} / \mathrm{dL}$, bilirrubina indirecta $3.68 \mathrm{mg} / \mathrm{dL}$.

Se midieron anticuerpos antinucleares (ANA), anticuerpos anticitoplasma (ANCA), IgG anticardiolipina, IgM anticardiolipina, anticuerpos anti-DNA doble cadena, anticuerpos anti-Smith y beta 2 microglobulina, el resultado fue no reactivo.

El 22 de octubre de 2019 egresó de Terapia Intensiva al Servicio de Medicina Interna. Se continuó tratamiento médico con prednisona vía oral calculada a $1 \mathrm{mg} / \mathrm{kg} /$ día e inmunoglobulina humana $0.4 \mathrm{~g} / \mathrm{kg} / \mathrm{día}$. Se reportó AST $11 \mathrm{UI} / \mathrm{L}$, ALT $48 \mathrm{UI} / \mathrm{L}$, bilirrubina total $1.63 \mathrm{mg} / \mathrm{dL}$, bilirrubina directa $0.69 \mathrm{mg} / \mathrm{dL}$, bilirrubina indirecta 0.94 $\mathrm{mg} / \mathrm{dL}$, leucocitos totales 3,400 células $/ \mathrm{mm}^{3}$, neutrófilos absolutos 2,900 células $/ \mathrm{mm}^{3}$, plaquetas 48,000 células $/ \mathrm{mm}^{3}$, hemoglobina $12.4 \mathrm{~g} / \mathrm{dL}$, hematocrito $35.2 \%$, Coombs directo positivo+.

El 25 de octubre del mismo año, se sospechó en piso de medicina interna vasculitis, se sugirió realizar biopsia de nervio tibial, la cual no procedió. El 26 de octubre de 2019, a pesar del tratamiento con hemodiálisis, presentó valores séricos de creatinina de $10.19 \mathrm{mg} / \mathrm{dL}$, urea de $209.90 \mathrm{mg} / \mathrm{dL}$, se realizó por parte del servicio de nefrología biopsia renal que reportó necrosis tubular intersticial.

El paciente fue egresado el 30 de octubre de 2019 a su domicilio, con seguimiento por parte de los Servicios de Nefrología y Hematología.

\section{RESULTADOS}

La evolución del paciente fue favorable al tratamiento médico de primera línea para el síndrome de FisherEvans, inmunoglobulina humana y esteroides (Figuras 2 y 3). Presentó mejoría de las cifras de hemoglobina, disminución de las cifras de bilirrubinas totales e incremento de la cuenta plaquetaria. Por último, fue egresado a domicilio con seguimiento en la consulta externa de nefrología y hematología. Se reportó por parte del servicio de nefrología recuperación de la función renal, con valores séricos de creatinina de $1.2 \mathrm{mg} / \mathrm{dL}$. 


\section{DISCUSIÓN}

El síndrome de Fisher-Evans es una entidad poco común. Se estima que de 0.8 al $4 \%$ de los pacientes con trombocitopenia inmunitaria o anemia hemolítica inmunitaria padecen este síndrome. ${ }^{7}$ La prevalencia de la enfermedad en México se desconoce. Se han descrito casos asociados a otras enfermedades inmunológicas ${ }^{3,4}$ en el ámbito mundial, pero no asociadas a pancreatitis. Senosiáin Labiano M y Alberdi Alonso publicaron en 2009 un reporte de caso de púrpura trombocitopénica inmunitaria asociada a pancreatitis. ${ }^{5}$

El paciente presentó a las 48 horas del diagnóstico de pancreatitis aguda anemia hemolítica inmunitaria, corroborada por la prueba de Coombs directo, hemoglobina de $7.2 \mathrm{mg} / \mathrm{dL}$, deshidrogenasa láctica 3,555 UI/L y púrpura trombocitopénica con plaquetas de 29,000 células $/ \mathrm{mm}^{3}$ y presencia de petequias en extremidades superiores; criterios diagnósticos del síndrome de Fisher-Evans. $^{1}$

Durante la estancia del paciente en la Unidad de Terapia Intensiva se sospechó falla hepática aguda por elevación de transaminasas, elevación de bilirrubinas totales y lesiones petequiales, consumo crónico de nuez vómica; sin embargo se descartó dicho diagnóstico por presencia de tiempos de coagulación dentro de parámetros normales.

Una vez realizado el diagnóstico de síndrome de Fisher-Evans, el paciente recibió tratamiento médico de primera línea. Recibió inmunoglobulina humana (0.4 $\mathrm{g} / \mathrm{kg} /$ día) durante cinco días, y metilprednisolona 500 mg IV cada 24 horas tres días, seguido por prednisona $1 \mathrm{mg} / \mathrm{kg} / \mathrm{día}$ vía oral, con respuesta favorable al tratamiento.

Con respecto a la lesión renal aguda, requirió sesiones de hemodiálisis, incluso a su egreso del hospital. Se realizó biopsia renal que reportó necrosis tubular in-

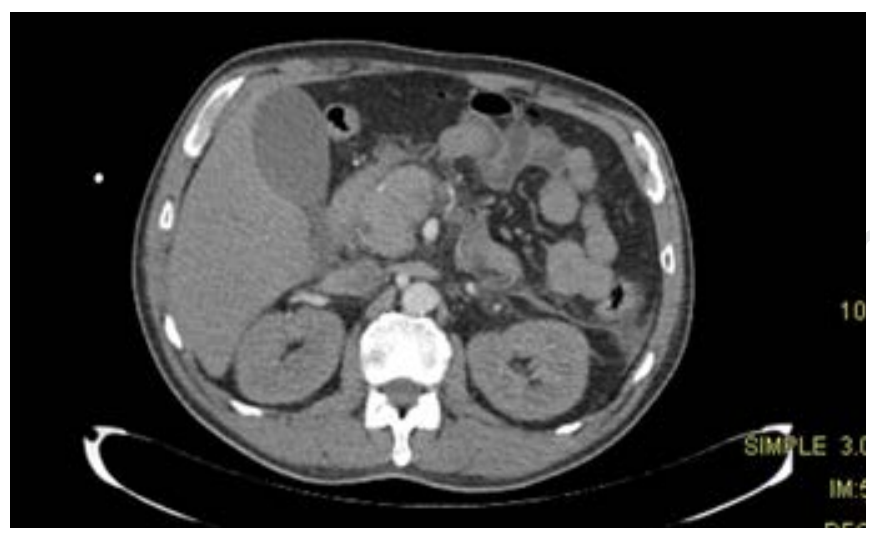

Figura 2: Imagen de tomografía axial computada general.

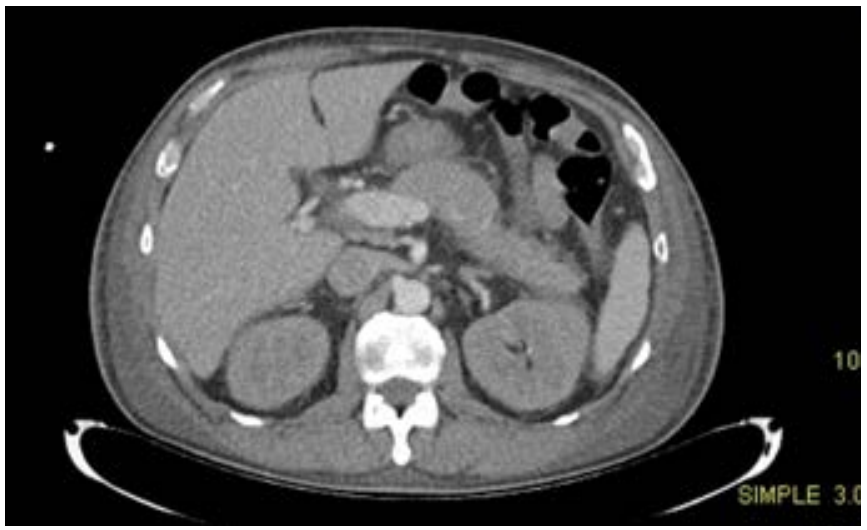

Figura 3: Imagen de tomografía axial computada que demuestra páncreas con mejoría.

tersticial con resolución de la función renal una semana después del egreso hospitalario.

\section{CONCLUSIÓN}

El síndrome de Fisher-Evans es una patología rara, asociada principalmente a otras enfermedades inmunológicas, existen factores asociados a la aparición de la enfermedad, en este caso pancreatitis aguda. La detección temprana de dicho síndrome y el manejo oportuno tuvo gran impacto en la evolución clínica del paciente.

\section{REFERENCIAS}

1. Jaime-Pérez J, Aguilar-Calderón P, Salazar-Cavazos L, GómezAlmaguer D. Evans syndrome: clinical perspectives, biological insights and treatment modalities. J Blood Med. 2018;9:171-184.

2. Cimá-Castañeda MA, Ayala-López PM, Lara-Palacios MI, AbblittLuengas SM, Jiménez-Báez MV. Síndrome de Fisher-Evans o de Evans. Rev Hematol Mex. 2016;17:144-149.

3. Silva RM, Urribastera CA, Fernández RL, Del Socorro $M$, Portelles MA. Síndrome de Fisher Evans asociado con esclerodermia. CCM. 2015; 19(3): 536-541.

4. Ayala E, Charaja K, Cruz I, Yupari M, Cornejo M, García M, et al. Síndrome de Evans en paciente con síndrome antifosfolipídico secundario: desafío terapéutico. Rev Cubana Reumatol. 2016;18(2):196-199.

5. Senosiáin LM, Lastra AP, Alberdi AJ. Púrpura trombótica trombocitopénica secundaria a pancreatitis aguda, una infrecuente asociación. Gastroenterología y Hepatología. 2009;32:531-532.

6. Norton A, Roberts I. Management of Evans syndrome. $\mathrm{Br} J$ Haematol. 2006;132(2):125-137.

Michel M, Chanet V, Dechartres A, Morin AS, Piette JC, Cirasino $L$, Rodeghiero $F$. The spectrum of Evans syndrome in adults: new insight into the disease based on the analysis of 68 cases. Blood. 2009;114(15):3167-3172.

Correspondencia:

Rafael Domínguez-Pérez

Calle Gabriel Mancera Núm. 222, Col. Del Valle, 03100, Alcaldía Benito Juárez, Ciudad de México, México.

Tel: 55 5639-5822, ext. 20761

E-mail: rafaeldp@hotmail.com 